\title{
Comparison of Fiber-Optic CDMA Systems Using Different Modulation Schemes
}

\author{
Pei-Cheng Chen, Bo-Yi Lu, Cheng-Yuan Chang* \\ Department of Electrical Engineering, National United University, Miaoli City 360, Taiwan, R.O.C. \\ *Corresponding Author: chengyuan@nuu.edu.tw
}

\begin{abstract}
In order to provide different data rate transmission and quality of service (QoS) for multimedia communications and networks, three common modulation techniques used for optical code-division multiple-access (O-CDMA) are multiple code (MC), multicode keying (MK) and pulse position modulation (PPM). In this paper, the performance and normalized spectral efficiency (NSE) of the O-CDMA systems using above three different modulation techniques are analyzed and compared. Moreover, the computer simulation of each O-CDMA system is performed and compared with the theoretical results to identify the validity of the performance analysis. Our numerical results show that MK scheme always has a better NSE than other two schemes (i.e., MC and PPM schemes), but PPM scheme can provide the best system performance among these three schemes at the expense of the increased bandwidth.
\end{abstract}

Keywords: optical code-division multiple-access, multicode keying, multiple code, pulse position modulation.

\section{Introduction}

Optical code-division multiple-access (O-CDMA) has been widely applied in optical communication systems and networks ${ }^{(1-3)}$, because the multiple access technique can provide many desirable features, such as dynamic bandwidth assignment, efficiency in bursty traffic, asynchronous and uncoordinated access, flexible user allocation and gradual performance degradation, compared with wavelength-division multiple-access (WDMA) and time-division multiple-access (TDMA) techniques ${ }^{(1-4)}$. O-CDMA can mainly allow many simultaneous users to share the same transmission channel by assigning a unique spreading code to each user ${ }^{(5)}$. Recently, with the growing demand for high speed networks and multimedia communication services, it is expected that the future O-CDMA systems should support a larger variety of multimedia services with different data rate and quality-of-service (QoS) to accommodate the transmission requirements of the system subscribers ${ }^{(1,6-8)}$. In addition to the multiple length and variable-weight techniques, three common modulation techniques which can support the different data rate and QoS transmission requirements for O-CDMA systems are multiple code (MC) ${ }^{(6)}$, multicode keying $(\mathrm{MK})^{(7,8)}$ and pulse position modulation $(\mathrm{PPM})^{(9,10)}$, respectively. In this paper, not only the performance but also normalized spectral efficiency (NSE) of three schemes are also analyzed and compared to determine which scheme should be used under certain conditions ${ }^{(8,11)}$.

The remainder of the paper is organized as follows. In Section 2, the system model of MC, MK and PPM schemes are studied. Numerical examples which evaluate and investigate the system parameters how to affect the system performance, are given in Section 3. Our results show that MK scheme always has a better NSE than MC and PPM schemes, but PPM scheme can provide the best performance among these three schemes at the expense of the increased bandwidth. Conclusions are finally drawn in Section 4.

\section{System Model}

First, a brief review of two-dimensional (2-D) multilevel prime code (MPC) $)^{(12)}$, which is used as numerical examples in the next section, is given in this section. Moreover, the system model of O-CDMA systems using MC, MK and PPM schemes are also introduced as follows.

\subsection{Multilevel Prime Code}

The use of $\left(L \times N, w, \lambda_{\mathrm{a}}=0, \lambda_{\mathrm{c}}=n\right)$ 2-D MPC ${ }^{(12)}$ with code cardinality $p^{n}$ (i.e., the number of codewords), the number of available wavelengths $L$, code length $N=p$ (i.e., number 
of time slots), code weight $w$, autocorrelation sidelobe of at most $\lambda_{\mathrm{a}}=0$, and cross-correlation value of at most $\lambda_{\mathrm{c}}=n$, where $p$ is a prime number and $n \leq w \leq p$, are assumed for the numerical examples in Section 3. Each code matrix of 2-D MPC can be denoted by $C_{i 1, i 2, \ldots, i n}=\left(c_{i 1, i 2, \ldots, i n, 0}, c_{i 1, i 2, \ldots, i n, 1}, \ldots\right.$, $\left.c_{i 1, i 2, \ldots, i n, j}, \ldots, c_{i 1, i 2, \ldots, i n, w-1}\right)$, in which the $j$ th element can be represented by ${ }^{(12)}$

$$
c_{i_{1}, i_{2}, \ldots, i_{n}, j}=\left(i_{1} j\right) \oplus_{p}\left(i_{2} j^{2}\right) \oplus_{p} \cdots \oplus_{p}\left(i_{n} j^{n}\right)
$$

which indicates the time-slot position (among $p$ ) of the $j$ th carrier wavelength for $j \in[0, w-1]$, where $\left\{i_{1}, i_{2}, \ldots, i_{n}\right\} \in$ $\mathrm{GF}(p)$, and “ $\oplus_{p}$ ” denotes a modulo- $p$ addition. Moreover, in order to obtain the larger cardinality in MC, MK, and PPM schemes, the use of the time-shifted copies of 2-D MPC is also assumed to represent the $m$ symbols ${ }^{(8,11,13)}$.

Using $N=p=5, L=w=5$, and $n=1$ as an example, based on (1), the $(5 \times 5,5,0,1) 2-\mathrm{D}$ MPC has five code matrices $C_{i 1}, i_{1} \in[0,4]$ (i.e., represented by $(0,0,0,0,0),(0,1,2,3,4)$, ( 0 , $2,4,1,3),(0,3,1,4,2),(0,4,3,2,1)$, respectively) of five timeshifted copies for each code matrix in Table 1. For example, five time-shifted copies of code matrix $C_{3}$ (i.e., $(0,3,1,4,2)$ ) are $(0,3,1,4,2),(1,4,2,0,3),(2,0,3,1,4),(3,1,4,2,0),(4,2,0,3$, $1)$ with time shift $\tau \in[0,4]$, respectively.

\subsection{Multiple Code Scheme ${ }^{(6,11)}$}

In the MC scheme, each user is allocated $m_{c}$ code matrices when a user need to transmit at the rate of $m_{c}$ times the basic bit transmission rate $R$ (i.e., the data bit rate of the on-off keying (OOK) scheme). First, the serial data bits are converted into the parallel data bits. Then, each parallel data bit one is transmitted with one of the allocated $m_{c}$ code matrices, but nothing is transmitted for a data bit zero ${ }^{(6,11)}$. That is, the number of code matrices allocated to each user determines the transmission bit rate of the scheme. Thus, the $m_{c}$ code matrices are transmitted at the same time results in date bit rate of the scheme $R_{\mathrm{MC}}=m_{c} \times R^{(11)}$. For example, the code matrix $C_{2}$ (i.e., $(0,2,4,1,3)$ ) and its three time-shifted copies (e.g., $(1,3,0,2,4),(3,0,2,4,1)$, and $(4,1,3$, $0,2)$ ), shown in Table 1 , are allocated to the user for $m_{c}=4$ multiple code transmission, thus the data bit rate of the scheme becomes $R_{\mathrm{MC}}=m_{c} \times R=4 R$.

\subsection{Multicode Keying Scheme (8,11) $^{(3)}$}

In this scheme, each user is assigned $m_{k}=2^{r}$ code matrices to represent $m_{k}$ symbols, corresponding to the transmission of $r$ data bits per symbol ${ }^{(8,11)}$. That is, every $r$ serial data bits are mapped into one symbol, and each symbol is transmitted with one of the assigned $m_{k}$ code matrices. Thus, the data bit rate of the scheme becomes $R_{\mathrm{MK}}=\left(\log _{2}\left\lfloor 2^{r}\right\rfloor\right) \times R$
Table 1. Code Matrices of $(5 \times 5,5,0, \lambda c=1) 2-D$ MPC and Its Time-Shifted Copies ${ }^{(12)}$

\begin{tabular}{c|c|c|c|c|c}
\cline { 2 - 6 } \multicolumn{5}{c}{ Level 1 } \\
\hline$\tau$ & $i_{1}=0$ & $i_{1}=1$ & $i_{1}=2$ & $i_{1}=3$ & $i_{1}=4$ \\
\hline 0 & $(0,0,0,0,0)$ & $(0,1,2,3,4)$ & $(0,2,4,1,3)$ & $(0,3,1,4,2)$ & $(0,4,3,2,1)$ \\
1 & $(1,1,1,1,1)$ & $(1,2,3,4,0)$ & $(1,3,0,2,4)$ & $(1,4,2,0,3)$ & $(1,0,4,3,2)$ \\
2 & $(2,2,2,2,2)$ & $(2,3,4,0,1)$ & $(2,4,1,3,0)$ & $(2,0,3,1,4)$ & $(2,1,0,4,3)$ \\
3 & $(3,3,3,3,3)$ & $(3,4,0,1,2)$ & $(3,0,2,4,1)$ & $(3,1,4,2,0)$ & $(3,2,1,0,4)$ \\
4 & $(4,4,4,4,4)$ & $(4,0,1,2,3)$ & $(4,1,3,0,2)$ & $(4,2,0,3,1)$ & $(4,3,2,1,0)$ \\
\hline
\end{tabular}

$=r R^{(8,11)}$, where $\lfloor\cdot\rfloor$ is the floor function. For example, the code matrix $C_{4}$ (i.e., $\left.(0,4,3,2,1)\right)$ and its three time-shifted copies (e.g., $(1,0,4,3,2),(2,1,0,4,3)$, and $(3,2,1,0,4))$, shown in Table 1 , are assigned to the user for $m_{k}=2^{2}=4$ multicode keying transmission, thus the data bit rate of the scheme becomes $R_{\mathrm{MK}}=\left(\log _{2}\lfloor 4\rfloor\right) \times R=2 R$.

\subsection{Pulse Position Modulation Scheme $e^{(9,10,14)}$}

Unlike MC or MK scheme, each user is only assigned one district code matrix in the PPM scheme, but the code matrix is located entirely in one of the $m_{p}=2^{s}$ PPM frames to represent the corresponding symbol, based on every transmitted $s$ data bits ${ }^{(9,10,14)}$, where $m_{p}=2^{s}$ is the number of PPM frames. Thus, the data bit rate of the scheme becomes $R_{\mathrm{PPM}}=\left(\log _{2}\left\lfloor 2^{s}\right\rfloor / 2^{s}\right) \times R=s R / 2^{s(9,10,14)}$. For example, the code matrix $C_{1}$ (i.e., $\left.(0,1,2,3,4)\right)$ is assigned to the user for $m_{p}=4$ PPM transmission. Then, the code matrix $C_{1}$ is placed into one of the $m_{p}=4$ PPM frames to represent one of $m_{p}=4$ symbols, based on every $s=2$ data bits, thus the data bit rate of the scheme becomes $R_{\mathrm{PPM}}=2 R / 2^{2}=R / 2$.

In summary, the above-mentioned three schemes can support the multi-rate transmission requirements by simply varying the number of the transmitted code matrices at the same time (i.e., $m_{c}$ ) for MC scheme, the number of bits per symbol (i.e., $\log _{2}\left\lfloor m_{k}\right\rfloor$ ) for MK scheme, and the number of PPM frames (i.e., $m_{p}$ ) for PPM scheme, respectively.

\section{Numerical Examples}

In this section, we mainly focus on the effect of multiple access interference (MAI) to system performance, and any physical noise, such as thermal noise, beat noise and shot noise are neglected ${ }^{(5,8,11,12)}$. It is because a practical OCDMA system should always perform with optical power above the noise level ${ }^{(11)}$. Moreover, the use of $(w \times p, w$, $\left.\lambda_{\mathrm{a}}=0, \lambda_{\mathrm{c}}=1\right)$ 2-D MPC briefly introduced in Section 2.1 is here assumed for the following numerical examples.

\subsection{Multiple Code Scheme}

The bit error probability of the MC scheme with the use of $(w \times p, w, 0,1)$ MPC for $m_{c}$ multiple code transmission is 
given by $^{(11)}$

$$
P_{e, \mathrm{MC}}(K)=\frac{1}{2} \sum_{i=0}^{w}(-1)^{w-i}\left(\begin{array}{l}
w \\
i
\end{array}\right)\left[q_{0}+q_{1} \frac{i}{w}\right]^{K m_{c}-1-h}
$$

where $K$ denotes the number of simultaneous users in the MC scheme and the effective number of interfering users is reduced to $K m_{c}-1-h$ and $h=m_{c}-1$ due to the use of $m_{c}$ timeshifted copies which have $\lambda_{\mathrm{c}}=0$. Moreover, $q_{j}$ denotes the probability of getting $j$ hits in a time slot, out of $\lambda_{\mathrm{c}}=1$ possible hits, $j \in[0,1]$, and are given by ${ }^{(11,12)}$

$$
\begin{gathered}
q_{1}=\frac{w}{2 p} \\
q_{0}=1-q_{1}=1-\frac{w}{2 p},
\end{gathered}
$$

respectively.

\subsection{Multicode Keying Scheme}

The bit error probability of the MK scheme with the use of $(w \times p, w, 0,1)$ MPC for $m_{k}$ multicode keying transmission is given by ${ }^{(8,11)}$

$$
P_{e, \mathrm{MK}}(K)=\frac{m_{k}}{2\left(m_{k}-1\right)}\left[1-\sum_{t=0}^{m_{k}-1} \frac{1}{t+1} P_{r}(w, t)\right]
$$

where $K$ denotes the number of simultaneous users in the MK scheme, and $P_{r}(w, t)$ represents the probability that $t$ decoders have an output intensity level of at least $w$ and $m_{k^{-}}$ 1- $t$ decoders have an output intensity level less than $w$ and is given by ${ }^{(8,11)}$

$$
P_{r}(w, t)=\left(\begin{array}{c}
m_{k}-1 \\
t
\end{array}\right)\left[P_{r}(w)\right]^{t}\left[\sum_{v=0}^{w-1} P_{r}(v)\right]^{m_{k}-1-t}
$$

where $P_{r}(v)$ denotes the probability of having a peak of $v$ appearing at any one of $m_{k}-1$ wrong decoder in the desired user and is obtained by ${ }^{(8,11)}$

$$
P_{r}(v)=\left(\begin{array}{l}
w \\
v
\end{array}\right) \sum_{i=0}^{v}(-1)^{i}\left(\begin{array}{l}
v \\
i
\end{array}\right)\left[q_{0}+q_{1} \frac{v-i}{w}\right]^{K-1}
$$

where the hit probabilities become $q_{1}=w / p$ and $q_{0}=1-w / p^{(11)}$, respectively, because of continuous symbol transmission, such that the factor $1 / 2$ is not need in MK scheme.

\subsection{Pulse Position Modulation Scheme}

The bit error probability of the PPM scheme using ( $w \times$ $p, w, 0,1)$ MPC for $m_{p}$ PPM transmission is modified from $(9,10,14)$ as

$$
\begin{aligned}
P_{e, \mathrm{PPM}}(K) & =\frac{m_{p}}{2\left(m_{p}-1\right)}\left[\left(m_{p}-1\right) \sum_{i=0}^{w}(-1)^{i}\left(\begin{array}{c}
w \\
i
\end{array}\right)\right. \\
& \left.\cdot\left[1-\frac{(i+w) w}{m_{p} w p}\right]^{K-1}-\left(\begin{array}{c}
\left(m_{p}-1\right) \\
2
\end{array}\right)\left[\frac{(K-1) w}{m_{p} w p}\right]^{2 w}\right](8)
\end{aligned}
$$

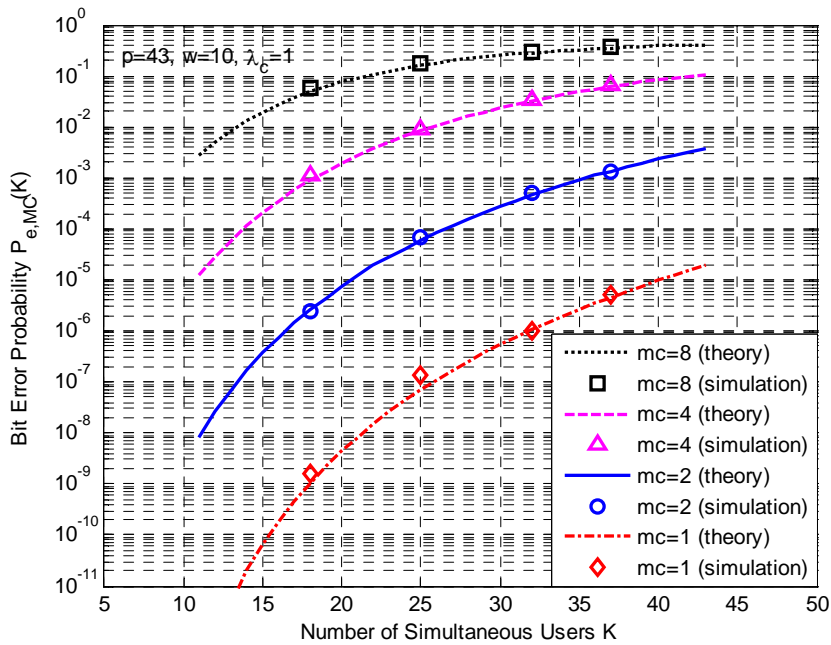

Fig. 1. Bit error probability $P_{\mathrm{e}, \mathrm{MC}}(K)$ of $\left(10 \times 43,10,0, \lambda_{\mathrm{c}}=1\right)$ MPC in MC scheme versus the number of simultaneous users $K$, where $m_{c}=\{1,2,4,8\}$.

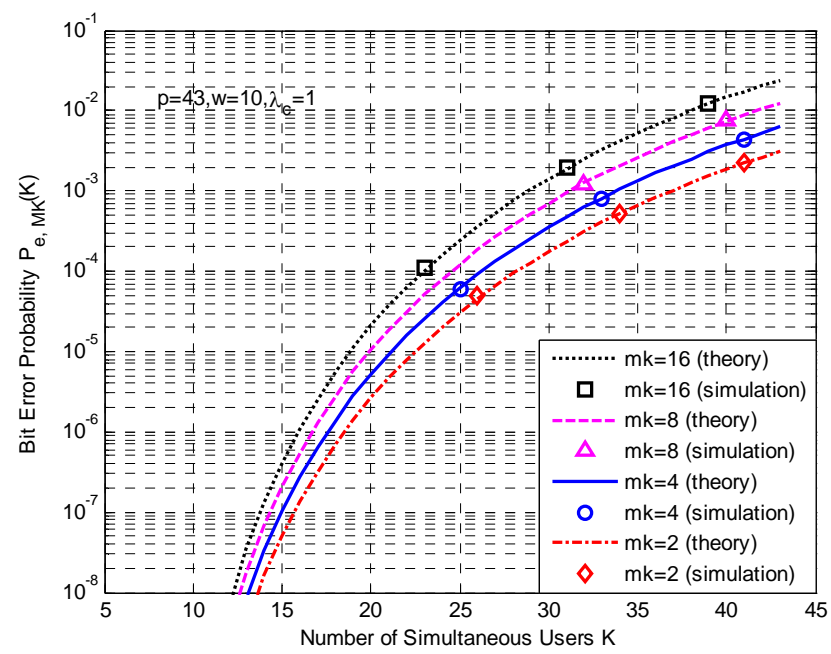

Fig. 2. Bit error probability $P_{\mathrm{e}, \mathrm{MK}}(K)$ of $\left(10 \times 43,10,0, \lambda_{\mathrm{c}}=1\right)$ MPC in MK scheme versus the number of simultaneous users $K$, where $m_{k}=\{2,4,8,16\}$.

where $K$ denotes the number of simultaneous users in the PPM scheme.

\subsection{Normalized Spectral Efficiency ${ }^{(8,11)}$}

In addition to the bit error probability, the another figure of merit, NSE, which considers the normalized data rate $R_{b}$ (i.e., set the basic bit transmission rate $R=1$ ), number of available wavelengths $L$, number of time slots $N$, and the number of simultaneous users $K$ as a whole, is used to fairly compare the three schemes and is given by ${ }^{(8,11)}$

$$
\text { NSE }=\frac{K R_{b}}{L N}
$$

The goal is to obtain the NSE as large as possible for better spectral efficiency or utilization.

In Fig. 1 , the bit error probabilities $P_{\mathrm{e}, \mathrm{MC}}(K)$ of the MC 


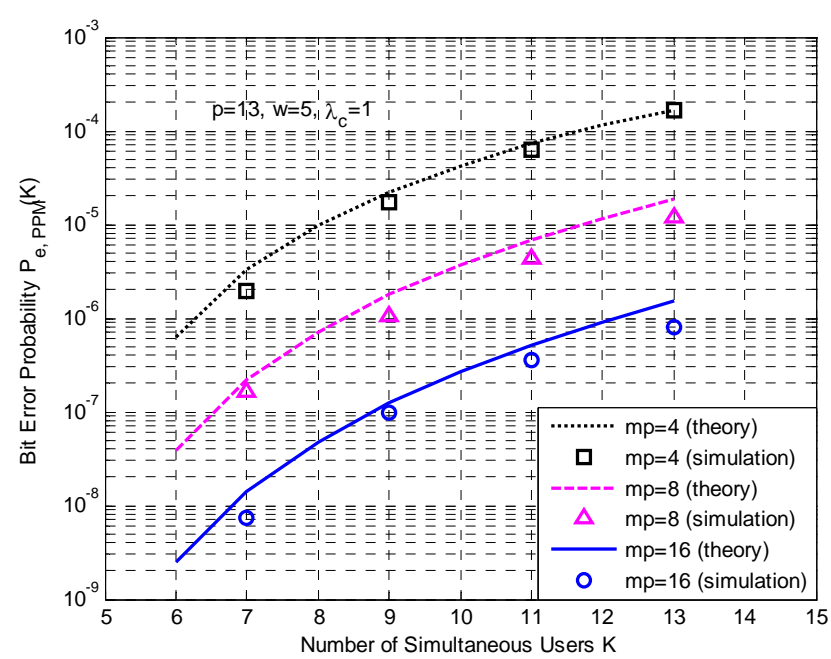

Fig. 3. Bit error probability $P_{\mathrm{e}, \mathrm{PPM}}(K)$ of $\left(5 \times 13,5,0, \lambda_{\mathrm{c}}=1\right)$ MPC in PPM scheme versus the number of simultaneous users $K$, where $m_{p}=\{4,8,16\}$.

scheme with $\left(10 \times 43,10,0, \lambda_{\mathrm{c}}=1\right) \mathrm{MPC}$, from (2), are plotted as a function of the number of simultaneous users $K$, where $m_{c}=\{1,2,4,8\}$. In general, the bit error probability gets worse as $K$ or $m_{c}$ increases, because there are more interfering code matrices which result in the effect of MAI, but the data bit rate of the MC scheme also increases as $m_{c}$ increases. That is, the MC scheme provides a trade-off between the system performance and date bit rate. Note that the dot-dashed curve with $m_{c}=1$ shows the performance of the OOK scheme, which agrees to ${ }^{(11, \text { eq. (12)) }}$. Also plotted in the figure are the computer-simulation results, which match closely with the theoretical results.

Fig. 2 shows the bit error probabilities $P_{\mathrm{e}, \mathrm{MK}}(K)$, from (5)-(7), of $\left(10 \times 43,10,0, \lambda_{\mathrm{c}}=1\right)$ MPC in MK scheme versus the number of simultaneous users $K$, where $m_{k}=\{2,4,8,16\}$. Similarly, the bit error probability gets worse as $K$ or $m_{k}$ increases, because there are more interfering code matrices, and there are more data bits to be decided incorrectly if a symbol detection error occurs. However, the MK scheme also can support the multi-rate transmission requirements by simply varying the number of bits per symbol (i.e., $\left.=\log _{2}\left\lfloor m_{k}\right\rfloor\right)$.

In Fig. 3, the bit error probabilities $P_{\mathrm{e}, \mathrm{PPM}}(K)$ of the PPM scheme using $\left(5 \times 13,5,0, \lambda_{\mathrm{c}}=1\right) \mathrm{MPC}$, from (8), are plotted against the number of simultaneous users $K$, where $m_{p}=\{4,8,16\}$. The bit error probability gets worse as $K$ increases but improves with $m_{p}$. It is because the hit probability will be reduced as the number of PPM frames (i.e., $m_{p}$ ) increases. In other words, the PPM scheme can mainly provide the better performance at the expense of the data bit rate and increased bandwidth. Again, both Figs. 2 and 3 also show the computer-simulation results, which match closely

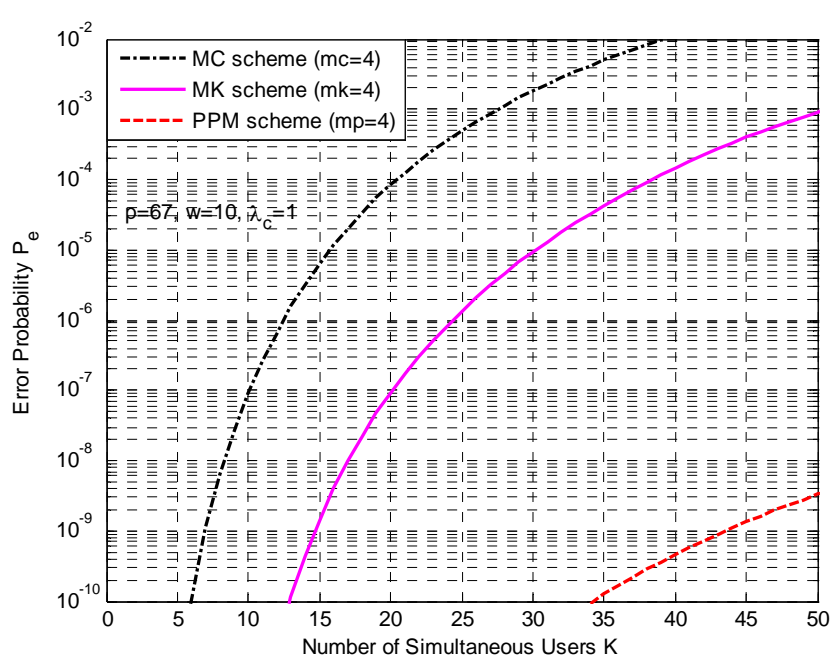

Fig. 4. Bit error probabilities of MC, MK and PPM schemes versus the number of simultaneous users $K$, with $(10 \times 67,10$, $\left.0, \lambda_{\mathrm{c}}=1\right)$ MPC, where $m_{c}=m_{k}=m_{p}=4$.

Table 2. Comparison of NSE for Three Schemes under Bit Error Probability $P_{\mathrm{e}}=10^{-9(8,11)}$.

\begin{tabular}{|c|c|c|c|c|c|c|}
\hline$P_{\mathrm{e}}=10^{-9}$ & $K$ & & $R_{b}$ & $L$ & $N$ & NSE \\
\hline MC & 6 & $m_{c}=4$ & 4 & 10 & 67 & 0.0358 \\
\hline MK & 14 & $m_{k}=4$ & 2 & 10 & 67 & 0.0418 \\
\hline PPM & 43 & $m_{p}=4$ & $1 / 2$ & 10 & 67 & 0.0321 \\
\hline
\end{tabular}

with the theoretical results.

Shown in Fig. 4 are the bit error probabilities $P_{\mathrm{e}}$ versus the number of simultaneous users $K$ plots of the MC, MK and PPM schemes with $\left(10 \times 67,10,0, \lambda_{\mathrm{c}}=1\right) \mathrm{MPC}$, where $m_{c}=$ $m_{k}=m_{p}=4$. Considering $P_{\mathrm{e}} \approx 10^{-9(8,11)}$, from the figure, we can also get the number of simultaneous users $K=6$ for the MC scheme, $K=14$ for the MK scheme, and $K=43$ for PPM scheme, respectively. Based on (9) and the data bit rate $R_{b}$ of three schemes, we then obtain NSE $=6 \times 4 /(10 \times 67)=$ 0.0358 for the MCscheme, $\mathrm{NSE}=14 \times 2 /(10 \times 67)=0.0418$ for the MK scheme, and NSE $=43 \times(1 / 2) /(10 \times 67)=0.0321$ for the PPM scheme, respectively.

Table 2 compares the NSEs of three schemes, based on Fig. 4. From the table, we can find that the MK scheme can provide the best spectral efficiency among these three schemes. On the other hand, from Fig. 4 and Table 2, we also can know that the PPM scheme can provide the best system performance among these three schemes, but the data bit rate of the PPM scheme is lower than those of the other two schemes. In other words, the PPM scheme provides the better performance at the expense of the data bit rate and increased bandwidth. 


\section{Conclusions}

In this paper, the three common modulation schemes (i.e., MC, MK and PPM schemes) which can support the different data rate and QoS transmission requirements for O-CDMA systems by simply varying the parameters of $m_{c}$, $m_{k}$ and $m_{p}$ are introduced and studied. Moreover, not only the performance but also the NSE of three schemes are also analyzed and compared to determine which scheme should be used under certain conditions. Our results show that the MK scheme always has a better NSE than other two schemes, but PPM scheme can provide the best system performance at the expense of the data bit rate or the increased bandwidth. Therefore, if system performance is more important than the spectral efficiency or data bit rate, one should use the PPM scheme. If the spectral efficiency is more important, one should use the MK scheme. That is, there is a trade-off between the system performance, data bit rate and spectral efficiency.

\section{Acknowledgment}

This work was supported by the Ministry of Science and Technology of Republic of China under Grant MOST 1032221-E-239-009.

\section{References}

(1) Hamzeh Beyranvand and Jawad A. Salehi: "Multirate and Multi-Quality-of-Service Passive Optical Network Based on Hybrid WDM/OCDM System”, IEEE Commun. Mag., Vol. 49, No. 2, pp. 39-44, 2011

(2) Ton Koonen: "Fiber to the Home/Fiber to the Premises: What, Where, and When?”, Proc. IEEE, Vol. 94, No. 5, pp. 911-934, 2006

(3) Alan E. Willner, Poorya Saghari, and Vahid R. Arbab: "Advanced Techniques to Increase the Number of Users and Bit Rate in OCDMA Networks”, IEEE J. Sel. Topics Quantum Electron., Vol. 13, No. 5, pp. 14031414, 2007

(4) S. Alireza Nezamalhosseini, Farokh Marvasti, Babak M. Ghaffari, and Jawad A. Salehi: "Interference Cancellation in Optical CDMA Systems via Advanced Binary Optical Logic Gate Elements”, IEEE Commun Mag., Vol. 50, No. 8, pp. 96-101, 2012

(5) Jawad A. Salehi and Charles A. Brackett: "Code Division Multiple-Access Techniques in Optical Fiber Networks-Part II: Systems Performance Analysis”, IEEE Trans. Commun., Vol. 37, No. 8, pp. 834-842, 1989
(6) Svetislav V. Maric and Vincent K.N. Lau: "Multirate Fiber-Optic CDMA: System Design and Performance Analysis”, J. Lightw. Technol., Vol. 16, No. 1, pp. 9-17, 1998

(7) Ngoc T. Dang and Anh T. Pham: "Reducing the Dispersion Effects in Multiwavelength Optical CDMA Systems by Using MCM Signaling”, J. Opt. Commun. Netw., Vol. 2, No. 11, pp. 967-974, 2010

(8) Cheng-Yuan Chang, Hung-Ta Chen, Guu-Chang Yang, and Wing C. Kwong: "Spectral Efficiency Study of QC-CHPCs in Multirate Optical CDMA Systems”, IEEE J. Select. Areas. Commun., Vol. 25, No. 9, pp. 118-128, 2007

(9) Hossam M.H Shalaby: "Performance Analysis of Optical Synchronous CDMA Communication Systems With PPM Signaling”, IEEE Trans. Commun., Vol. 43, No. 4, pp. 624-634, 1995

(10) Hossam M.H Shalaby: "Direct Detection Optical Overlapping PPM-CDMA Communication Systems With Double Optical Hardlimiters”, J. Lightw. Technol., Vol. 17, No. 7, pp. 1158-1165, 1999

(11)Hung-Wei Chen, Guu-Chang Yang, Cheng-Yuan Chang, Tsung-Chi Lin, and Wing C. Kwong: "Spectral Efficiency Study of Two Multirate Schemes for Asynchronous Optical CDMA”, J. Lightw. Technol., Vol. 27, No. 14, pp. 2771-2778, 2009

(12) Chieh-Hung Hsieh, Guu-Chang Yang, Cheng-Yuan Chang, and Wing C. Kwong: "Multilevel Prime Codes for Optical CDMA Systems”, J. Opt. Commun. Netw., Vol. 1, No. 7, pp. 600-607, 2009

(13) Evgenii Narimanov, Wing C. Kwong, Guu-Chang Yang, and Paul R. Prucnal: "Shifted Carrier-Hopping Prime Codes for Multicode Keying in WavelengthTime O-CDMA”, IEEE Trans. Commun., Vol. 53, No. 12, pp. 2150-2156, 2005

(14) Anderson L. Sanches, José Valdemir dos Reis, Jr. and Ben-Hur V. Borges: “Analysis of High-Speed Optical Wavelength/Time CDMA Networks Using Pulse-Position Modulation and Forward Error Correction Techniques”, J. Lightw. Technol., Vol. 27, No. 22, pp. 5134-5144, 2009 